\title{
Persistent Adherence to Airway Clearance Therapy in Adults With Cystic Fibrosis
}

\author{
Allen C Sherman, Stephanie Simonton-Atchley, Dianne Campbell, Raghu M Reddy, \\ Catherine E O'Brien, Bethany Guinee, Laura D Wagner, and Paula J Anderson
}

\begin{abstract}
BACKGROUND: Airway clearance therapy (ACT) is a core component of daily treatment for cystic fibrosis (CF). However, surprisingly little is known about sustained or persistent use of ACT over time among adults with CF. This longitudinal study examined persistent adherence to ACT over 12 months and its modifiable predictors, drawing on aspects of Social Cognitive Theory and the Theory of Planned Behavior. METHODS: Subjects were drawn from a regional CF center in the southern United States. Predictor variables evaluated at baseline included self-efficacy for ACT (ie, self-confidence in overcoming barriers), outcome expectations (ie, perceived necessity of ACT and concerns about its disruptive effects), and subjective norms (ie, perceptions of being influenced by others). The Cystic Fibrosis Treatment Questionnaire (CFTQ) was used to assess self-reported adherence to ACT at baseline, at 6 months, and at 12 months. RESULTS: The mean age of subjects was $27.2 \pm 9.1 \mathrm{y}$, and mean $\mathrm{FEV}_{1} \%$ predicted was $65.5 \pm 24.8$. Forty-six percent of subjects reported persistent use of ACT (classified as adherent at all assessment periods). In bivariate analyses, all social cognitive predictor variables assessed at baseline were significantly related to persistent adherence (all $P<.03$ ), except subjective norms. In logistic regression analyses that modeled the effects of these predictors simultaneously while controlling for $F E V_{1} \%$, fewer baseline concerns about ACT (odds ratio $=0.82,95 \%$ CI 0.69-0.99) and greater self-efficacy (odds ratio $=1.09,95 \%$ CI 1.01-1.18) remained significant independent predictors. CONCLUSIONS: This longitudinal study addresses an important gap in the literature regarding adherence to ACT over time $(12 \mathrm{mo})$ in a routine clinical setting. Persistent adherence was problematic. As anticipated, social cognitive variables (self-confidence and perceived concerns) predicted self-reported persistence, and these may represent practical targets for intervention. Key words: adherence; persistence; airway clearance therapy; cystic fibrosis; social cognitive theory. [Respir Care 2019;64(7):778-785. (C) 2019 Daedalus Enterprises]
\end{abstract}

\section{Introduction}

Despite advances in treatment, individuals with cystic fibrosis $(\mathrm{CF})$ are confronted with considerable chal- lenges including pulmonary exacerbations, infectious and diabetic complications, and shortened life expectancy. Survival rates have improved appreciably, but treatment regimens are complex and highly burdensome.

\footnotetext{
Drs Sherman, Simonton-Atchley, and Ms Wagner are affiliated with the Behavioral Medicine Division, University of Arkansas for Medical Sciences, Little Rock, Arkansas. Dr Anderson is affiliated with the Department of Internal Medicine, University of Arkansas for Medical Sciences, Little Rock, Arkansas. Drs O'Brien and Guinee are affiliated with the Department of Pharmacy Practice, University of Arkansas for Medical Sciences, Little Rock, Arkansas. Dr Reddy is affiliated with St. Vincent's Hospital, Little Rock, Arkansas. Ms Campbell is affliated with Social Work Services at the Central Arkanas Veteran Healthcare System, Little Rock, Arkansas.
}

At the time of this study, Ms Campbell was affiliated with the Cystic Fibrosis Clinic, University of Arkansas for Medical Sciences, and Dr Reddy was affiliated with the Department of Internal Medicine, University of Arkansas for Medical Sciences, Little Rock, Arkansas.

Based in part on data presented at the Annual Meeting of the American Psychosomatic Society held on March 11, 2016 , in Denver, Colorado by Dr Sherman.

The authors have disclosed no conflicts of interest. 
A core component of treatment for most subjects is airway clearance therapy (ACT) such as chest physiotherapy, positive expiratory pressure, high-frequency chest wall oscillation, and postural drainage and percussion, which is commonly used two or more times per day to enhance mucus transport and reduce bacterial load. ${ }^{1}$ These approaches are part of a broader tapestry of treatment that includes pancreatic enzymes and nutritional therapies, oral or nebulized antibiotics, nebulized mucolytics and osmotic agents, and exercise. Not surprisingly, treatment adherence presents a formidable challenge- barriers have been amply catalogued by both quantitative ${ }^{2,3}$ and qualitative ${ }^{4}$ investigations. Generally, adherence to ACT is poorer relative to most medications or enzyme supplements..$^{5-7}$

Although concerns about use of ACT have long been recognized, surprisingly little is known about continued adherence over time (ie, persistence). Most studies of adults have used cross-sectional designs, offering few hints about longer-term adherence, which is critical information. Data from randomized clinical trials, which included both children and adults, suggested that average levels of self-reported adherence were $<40 \%$ at baseline, and averaged only roughly $50-60 \%$ over the course of the intervention. ${ }^{8,9}$ Additional information is needed about ongoing or persistent adherence to ACT in routine care settings, outside the limited context of clinical trials.

Another central priority for $\mathrm{CF}$ treatment centers is to understand the factors that influence ACT adherence or persistence. Clinical variables such as disease severity have not been reliable predictors for adults with $\mathrm{CF},{ }^{5-8,10,11}$ nor have demographic characteristics $5,7,11,12$ or subjects' knowledge of the illness. $5,6,12$

Some investigators have begun to evaluate psychosocial influences. ${ }^{10,13}$ Social cognitive theory provides a useful conceptual framework. ${ }^{14}$ This model emphasizes the importance of self-efficacy (ie, confidence in one's ability to implement a specific behavior) and outcome expectations (ie, beliefs about the consequences of these actions). In other illnesses (eg, coronary heart disease, HIV disease, diabetes), increased self-efficacy has been tied to enhanced medication adherence. ${ }^{15,16}$ More recently, outcome expectations regarding the perceived necessity of treatment, and concerns about its adverse consequences, have been linked with adherence as well. ${ }^{17,18}$ Investigators have begun to examine whether some of these variables might be tied to CF treatment

Correspondence: Allen C Sherman PhD, Behavioral Medicine, \#756, 4301 W Markham St, Little Rock, AR 72205. E-mail: shermanallenc@uams.edu.

DOI: $10.4187 /$ respcare. 06500

\section{QUICK LOOK}

\section{Current knowledge}

Airway clearance therapy (ACT) is a critical aspect of care for cystic fibrosis (CF), but it is widely recognized that home adherence is problematic among adults with CF. Previous studies of adherence have relied on cross-sectional designs that involve a single assessment period. In other chronic illnesses, modifiable risk factors for poor adherence include social cognitive variables (eg, self-efficacy, outcome expectations).

\section{What this paper contributes to our knowledge}

Self-reported persistent adherence to ACT among adults with $\mathrm{CF}$ treated in a natural clinical setting was poor. These findings offer a fuller picture of adherence by examining use over the course of a year. Greater self-efficacy or self-confidence for overcoming barriers to ACT use, and fewer concerns about its disruptive or harmful effects, predicted better persistent adherence to ACT. These results point to practical, modifiable targets that might be addressed to improve persistent adherence among adults with CF.

adherence, ${ }^{19,20}$ but their associations with adherence to ACT among adults is unknown. According to the Theory of Planned Behavior, another relevant factor might involve subjective norms, or perceptions of being influenced by other people to engage in a particular health practice. ${ }^{21}$ These too have yet to be investigated among adults with CF. Each of these theoretical factors represents attractive targets for investigation because they are modifiable rather than non-modifiable.

The current study was a longitudinal investigation examining self-reported adherence to ACT among adult subjects with CF over the course of 12 months. We were particularly interested in levels of persistence, or sustained adherence over time (aim 1). An additional aim was to examine modifiable, theoretically-based factors associated with persistent adherence (aim 2). We hypothesized that greater baseline self-efficacy or selfconfidence in use of ACT, fewer concerns about its adverse consequences, greater perceptions of treatment necessity, and stronger perceived social norms supporting its use, would each be predictive of greater persistent adherence, controlling for any significant clinical or demographic factors. In addition, exploratory analyses were used to assess relationships between persistence and other potential predictive factors sometimes noted in the literature, including baseline depressive symptoms and social support. 


\section{Methods}

\section{Subjects}

Subjects were followed at a regional adult CF clinic at the University of Arkansas for Medical Sciences. All adult out-patients with CF treated by the multidisciplinary care team were eligible for this longitudinal study. Exclusion criteria included age $<18$ y or functional status sufficiently compromised to preclude completion of questionnaires (no subjects met this criterion). The study was approved by the Institutional Review Board. Subjects were approached during routine clinic visits, provided written consent, and completed a packet of questionnaires between January 2011 and October 2013. At 6-month and 12-month follow-ups, they completed another packet of questionnaires in the clinic, or via mail if they were not in clinic at that time. Subjects who did not return the packet received a minimum of 2 reminders by telephone or mail.

\section{Adherence Outcomes}

Persistent Adherence Over Time. At each of the assessment periods, self-reported adherence to ACT was evaluated with the Cystic Fibrosis Treatment Questionnaire ${ }^{20}$ (CFTQ), which is based on an earlier version ${ }^{5}$ and measures adherence using a 5-point scale. The CFTQ seeks to minimize demand characteristics and inflated reporting by explicitly acknowledging that many subjects tailor their adherence to meet individual needs. The measure has demonstrated good internal consistency and construct validity among subjects with CF.7,20 The primary outcome was persistent adherence to ACT across all assessment periods, as indicated by an item that evaluated frequency of treatment at each assessment. Response options include $1=$ never, $2=\mathrm{I}$ often missed sessions, $3=\mathrm{I}$ missed $>2$ sessions each week, $4=\mathrm{I}$ only missed once or twice per week, and $5=$ I almost always do my physiotherapy or airway therapy. Responses were dichotomized; subjects who reported always doing treatment or missing no more than once or twice per week were classified as adherent at that assessment period, consistent with prior research. ${ }^{5,6,10,13}$ All others were classified as nonadherent. "Persistent" or sustained adherence was defined as adherence at all assessment periods over the course of the year; "nonpersistence" denoted subjects who were classified as nonadherent during one or more assessment periods.

Adherence at 12 Months. Subjects also completed the Medication Adherence Report Scale (MARS) at each assessment, which includes 9 items that assess ACT nonadherence, to which subjects respond on a 5-point scale. ${ }^{22}$ Like the CFTQ, MARS was designed to reduce demand characteristic through the use of sensitively phrased items.
Evidence has supported its internal consistency and construct validity. ${ }^{22}$ As expected, scores on the CFTQ and MARS ACT scales were highly correlated $(r=.70)$, so they were combined. Following Bucks et al, ${ }^{20}$ we reversed the scoring on the MARS so that higher scores represented greater adherence, standardized the items on these 2 measures, and summed them to create a composite score (a continuous variable), which was used as a secondary outcome. Cronbach's alpha for the composite measure was .95 .

\section{Predictors of Adherence}

Self Efficacy. An 11-item instrument derived for this study (Self-Efficacy for Airway Clearance) was used to assess self-efficacy for adhering to ACT, because no validated measures were available. Its conceptual foundation was based on reasons for nonadherence previously documented in a large study of adult subjects with $\mathrm{CF}^{7}{ }^{7} \mathrm{We}$ selected items from each of the 4 empirically derived factors identified in that investigation (ie, emotional consequences of treatment, treatment does not help, fitting treatment into lifestyle, and physical consequences of treatment). The measure asks subjects to rate their confidence in adhering to ACT in response to each of these challenges. Subjects respond on a 5 -point scale $(1=$ not at all confident, $5=$ extremely confident). The baseline assessment was used in the analyses. Cronbach's alpha was .96. To assess convergent validity, a subgroup of subjects completed another validated measure of self-efficacy for adherence, the SelfEfficacy for Appropriate Medication Use Scale (SEAMS) at the 12-month assessment. ${ }^{16}$ This measure was added to the packet after the study had begun, and it was used with the last 23 consecutive subjects. Because the SEAMS instrument was designed to assess adherence to medication, it was modified to refer to chest physiotherapy or airway clearance instead. The correlations of the baseline $(r=.54$, $P=.008)$ and 6 -month $(r=.69, P<.001)$ scores from the Self-Efficacy for Airway Clearance instrument with the 12-month SEAMS scores were high, supporting the convergent validity of our measure of self-efficacy.

Outcome Expectations. Expectations regarding ACT were assessed at baseline using the Beliefs about Medications Questionnaire-Specific ${ }^{22}$ (BMQ-Specific). This instrument includes 2 subscales that assess beliefs about the necessity of specific treatments (ie, ACT) and concerns about harmful or disruptive effects of specific treatments. Subjects respond to each item on a 5-point scale $(1=$ strongly disagree, $5=$ strongly agree $)$. The instrument has demonstrated good internal consistency and construct validity in samples of medical subjects. ${ }^{22}$ The measure is intended to be modified for different treatment contexts; we used the version developed by Bucks et al, ${ }^{20}$ 
which targeted ACT. Cronbach's alpha in the current sample was 0.87 for perceived necessity and 0.66 for concerns. Additionally, subjective norms regarding adherence were assessed using an item adapted from Courneya and Friedenreich $^{23}$ (ie, "People who are important to me think that I should practice airway clearance/chest physiotherapy regularly."). Response options ranged from 1 = "strongly disagree" to 5 = "strongly agree."

Social Desirability. As a check on social desirability response bias, subjects completed the abbreviated 13-item Marlowe-Crowne Social Desirability Scale at baseline, which has demonstrated good factor structure and convergent validity. ${ }^{24}$

Other Predictor Variables. At baseline, depressive symptoms were evaluated using the Hospital Anxiety and Depression Scale (HADS), a widely used measure whose psychometric properties are well documented. ${ }^{25}$ Perceived social support was evaluated with the MOS Family Functioning Scale, a 4-item measure of satisfaction with family life, which has demonstrated good internal consistency and construct validity ${ }^{26}$; negative or undermining social interactions were evaluated using the 5-item version of the Social Constraints Scale, whose construct validity is wellestablished. ${ }^{27}$

Demographic and Clinical Variables. Subjects completed a brief demographic form (eg, age, education). Clinical variables were abstracted from the medical record (eg, $\mathrm{FEV}_{1} \%$ predicted, body mass index, lung transplant status, comorbidities, number of hospital admissions in past $12 \mathrm{mo})$.

\section{Statistical Analyses}

Descriptive statistics were used to summarize scores on the ACT adherence indices (study aim 1). Preliminary bivariate analyses evaluated associations between persistent/sustained adherence (ie, adherent at all assessment periods vs nonadherent at any assessment) and baseline demographic/clinical characteristics (eg, age, gender, education, marital status, income, age at diagnosis, $\mathrm{FEV}_{1} \%$ predicted, body mass index, number of comorbidities, number of hospitalizations in the past year) using $t$ tests or chi-square analyses. Log transformations were used for current age and age at diagnosis due to nonnormal distributions. Respiratory function ( $\mathrm{FEV}_{1} \%$ predicted) was used as an indicator of disease severity. In the primary analyses, a multivariable logistic regression model was used to test hypothesized associations between baseline social cognitive predictor variables (ie, self-efficacy for ACT, perceived concerns about ACT, perceived necessity, and subjective norms) and subsequent persistence, controlling for baseline disease severity and any clinical or demographic variables that were significant in the initial bivariate analyses (study aim 2). Subjective norms were dichotomized (median split) due to unsuccessful efforts to transform the skewed distribution. Tests were 2 -tailed, and $P<.05$ were considered significant. In sensitivity analyses, the primary analysis was repeated controlling for social desirability response bias instead of disease severity.

In the secondary analyses, we examined the effects of baseline social cognitive predictors on the 12-month adherence composite score (ie, combined CFQT and MARS subscales, a continuous variable), using multiple regression analyses and adjusting for baseline disease severity. Finally, exploratory analyses evaluated bivariate associations of other predictor variables (ie, baseline depressive symptoms, social support) with persistent adherence over time, using $t$ tests.

For subjects who died before or did not return the 12month assessment ( $n=5$ subjects), the analyses used adherence data from the previous (6-mo) assessment if available to address these missing values. Sensitivity analyses were conducted to examine findings when these subjects were excluded from the models $(n=61$ subjects).

\section{Results}

Of 84 subjects invited to participate, 78 completed consent forms and the baseline assessment packet $(92 \%$ accrual rate). Data from 2 subjects were deleted from the analyses due to extensive missing values and random responding. Among the 76 subjects at baseline, 3 were not prescribed ACT at baseline, 5 dropped out, and an additional 12 subjects either died before returning $(n=4)$ or did not return one of the follow-up evaluations despite reminders $(n=8)$, leaving a final evaluable sample of 66 subjects with data from at least 2 assessments. More specifically, 61 subjects were assessed regarding ACT at 6 months, and 62 subjects were assessed at 12 months. Attrition analysis indicated that dropouts had significantly lower levels of education than completers $(P<.001)$; there were no significant differences regarding other demographic or clinical variables or adherence.

\section{Self-Reported Persistent Adherence to ACT}

Sample characteristics are summarized in Table 1. Mean age was $27.15 \pm 9.05 \mathrm{y}$, mean $\mathrm{FEV}_{1} \%$ predicted was $65.47 \pm 24.82$, and $54.6 \%$ of the subjects were male. Persistent adherence across all available assessments over time was reported by 30 subjects $(45.5 \%)$; 36 subjects $(54.5 \%)$ demonstrated poor persistence. (The proportion of subjects classified as adherent at each time point was $56.1 \%$ at baseline, $61.7 \%$ at $6 \mathrm{mo}$, and $65.6 \%$ at $12 \mathrm{mo}$.) 
Table 1. Sample Characteristics

\begin{tabular}{|c|c|}
\hline Characteristic & Value \\
\hline Age, y & $27.15 \pm 9.05$ \\
\hline Education, y & $13.35 \pm 1.97$ \\
\hline $\mathrm{FEV}_{1} \%$ predicted & $65.47 \pm 24.82$ \\
\hline Body mass index, $\mathrm{kg} / \mathrm{m}^{2}$ & $21.89 \pm 2.98$ \\
\hline Hospitalizations past year & $0.83 \pm 1.16$ \\
\hline Male, $n(\%)$ & $36(54.6)$ \\
\hline Married/cohabitating, $n(\%)$ & $17(25.8)$ \\
\hline \multicolumn{2}{|l|}{ Ethnicity, $n(\%)$} \\
\hline White & $63(95.5)$ \\
\hline Non-white & $3(4.6)$ \\
\hline \multicolumn{2}{|l|}{ Income, $n(\%)$} \\
\hline$\$ 0-19,999$ & $30(45.5)$ \\
\hline$\geq \$ 20,000$ & $36(55.6)$ \\
\hline \multicolumn{2}{|l|}{ Employment, $n(\%)$} \\
\hline Full or part-time work & $31(47.0)$ \\
\hline Disabled & $22(33.3)$ \\
\hline Student & $7(10.6)$ \\
\hline Homemaker & $3(4.5)$ \\
\hline Unemployed & $3(4.5)$ \\
\hline \multicolumn{2}{|l|}{ Persistent Adherence, $n(\%)$} \\
\hline High & $30(45.5)$ \\
\hline Low & $36(54.5)$ \\
\hline 12-month adherence composite (possible range: $13-65$ ) & $50.94 \pm 12.36$ \\
\hline \multicolumn{2}{|l|}{ Social cognitive variables } \\
\hline Self-efficacy (possible range: $11-55$ ) & $42.24 \pm 10.65$ \\
\hline Perceived concerns (possible range: $6-30$ ) & $11.65 \pm 4.11$ \\
\hline Perceived necessity (possible range: $5-25$ ) & $19.12 \pm 4.82$ \\
\hline Subjective norms (possible range: $1-5$ ) & $4.65 \pm 0.93$ \\
\hline HADS depression (possible range: $0-21$ ) & $3.58 \pm 4.08$ \\
\hline $\begin{array}{l}\text { Data are presented as } n(\%) \text { or mean } \pm \text { SD. } \\
\text { HADS }=\text { Hospital Anxiety and Depression Scale }\end{array}$ & \\
\hline
\end{tabular}

On average, subjects reported moderately high levels of self-confidence for adherence to ACT (item $\mathrm{M}=3.8$ on a 5-point scale), moderately strong beliefs about its necessity (item $\mathrm{M}=3.8$ on a 5-point scale), moderate concerns about its use (item $\mathrm{M}=2.3$ on a 5-point scale), and strong perceptions that other people in their lives wanted them to adhere to ACT (item $\mathrm{M}=4.7$ on a 5-point scale).

\section{Predicting Persistent Adherence from Social Cognitive Variables}

In initial bivariate analyses, self-reported persistent adherence over time was not associated with social desirability response bias (ie, efforts to present a favorable impression, $P=.11$ ), nor was it associated with any of the baseline clinical (eg, hospitalizations, body mass index, comorbidities) or demographic (eg, age, education, marital status, income) variables assessed (all $P>.22$ ).

As expected, in bivariate analyses, persistent adherence was significantly associated with greater baseline self-ef-
Table 2. Bivariate Associations Between Social Cognitive Variables at Baseline and Persistent Adherence to Airway Clearance Therapy

\begin{tabular}{|c|c|c|c|c|}
\hline Predictor & $\begin{array}{c}\text { High } \\
\text { Persistence }\end{array}$ & $\begin{array}{c}\text { Low } \\
\text { Persistence }\end{array}$ & $P$ & $\begin{array}{l}\text { Effect } \\
\text { Size, d }\end{array}$ \\
\hline Self-efficacy & $47.47 \pm 8.82$ & $37.89 \pm 10.17$ & $<.001 *$ & 1.01 \\
\hline Concerns & $9.80 \pm 3.99$ & $13.19 \pm 3.58$ & $<.001 *$ & 0.89 \\
\hline Necessity & $20.60 \pm 4.41$ & $17.89 \pm 4.85$ & $.02 *$ & 0.59 \\
\hline \multicolumn{5}{|c|}{ Subjective norms } \\
\hline Low & $3(10.3)$ & $9(25.0)$ & $.20 \dagger$ & \\
\hline High & $26(89.7)$ & $27(75.0)$ & & \\
\hline \multicolumn{5}{|c|}{$\begin{array}{l}\text { Data are presented as } \mathrm{n}(\%) \text { or mean } \pm \mathrm{SD} \text {. } \\
* P \text { value derived from } t \text { test. } \\
\dagger P \text { value derived from Fisher exact test. }\end{array}$} \\
\hline
\end{tabular}

ficacy for ACT $(P<.001)$, fewer concerns about this treatment $(P<.001)$, and greater perceived necessity $(P=.02$; see Table 2$)$. It was not related to subjective norms for use of ACT $(P=.20)$. In multivariable logistic regression analyses, which included all social cognitive variables and controlled for baseline disease severity ( $\mathrm{FEV}_{1} \%$ predicted), persistence was predicted by fewer baseline concerns about ACT (odds ratio $=0.82,95 \% \mathrm{CI}$ $0.69-0.99$ ) and higher baseline self-efficacy (odds ratio $=1.09,95 \%$ CI 1.01-1.18). Findings were similar in sensitivity analyses which controlled for social desirability instead of disease severity (concerns: odds ratio $=0.82,95 \%$ CI $0.68-0.99$; self-efficacy: odds ratio $=1.09,95 \%$ CI 1.01-1.18), and in sensitivity analyses that included only subjects with data at the 12-month assessment ( $n=61$ ), except that perceived concerns became marginally significant (concerns: odds ratio $=0.84,95 \%$ CI $0.70-1.01$; self-efficacy: odds ratio $=1.11,95 \%$ CI 1.02-1.21).

\section{Predicting Adherence at 12 Months from Social Cognitive Variables}

In bivariate analyses, as anticipated, the adherence composite score (CFTQ and MARS) at 12 months was significantly associated with greater baseline self-efficacy for ACT $(r=.52, P<.001)$, fewer concerns about ACT $(r=-0.40, P<.001)$, stronger perceived necessity ( $r=.40, P<.001)$, and stronger subjective norms $(P=.01)$. In multiple regression analyses that included all social cognitive variables in the model and controlled for baseline disease severity, the 12-month adherence score was significantly predicted by greater baseline self-efficacy for ACT $(\beta=.32, P=.026)$ and marginally significantly related to fewer baseline concerns $(\beta=-0.21, P=.081)$. 
Table 3. Bivariate Associations Between Other Predictor Variables at Baseline and Persistent Adherence to Airway Clearance Therapy

\begin{tabular}{lrrrc}
\hline \hline \multicolumn{1}{c}{ Predictor } & \multicolumn{1}{c}{$\begin{array}{c}\text { High } \\
\text { Persistence }\end{array}$} & \multicolumn{1}{c}{$\begin{array}{c}\text { Low } \\
\text { Persistence }\end{array}$} & $P$ & $\begin{array}{c}\text { Effect } \\
\text { Size, d }\end{array}$ \\
\hline Depressive symptoms (log) & $1.11 \pm 0.13$ & $1.12 \pm 0.11$ & .75 & 0.08 \\
MOS Family support & $18.28 \pm 3.91$ & $16.47 \pm 3.86$ & .07 & 0.46 \\
Social constraints (log) & $0.93 \pm 0.17$ & $1.01 \pm 0.22$ & .10 & 0.43
\end{tabular}

$P$ value derived from $t$ test.

MOS $=$ Medical Outcomes Survey

\section{Associations of Other Variables with Persistent Adherence}

In exploratory bivariate analyses, there were marginally significant effects for social support variables (Table 3): subjects who experienced stronger family support $(P=.07)$ and fewer negative or undermining relationships $(P=.10)$ demonstrated a nonsignificant trend for greater persistence over time. There were no significant associations of persistent adherence with baseline depressive symptoms $(P>$.74).

\section{Discussion}

To our knowledge, this investigation is the first longitudinal study to examine self-reported sustained adherence (ie, "persistence") to ACT over time, rather than only concurrently, among adults receiving routine care for CF. Moreover, it is the first to scrutinize the predictive role of treatment-specific self-efficacy (ie, self-confidence for overcoming barriers) and outcome expectations (ie, anticipated consequences) for ACT among these adults. Findings suggested low levels of adherence at each assessment period and, more critically, low levels of persistent adherence over the course of the study (45\%). No data are available from other naturalistic studies to allow for comparisons regarding persistent adherence. With respect to the point-prevalence of self-reported adherence to ACT (at a single time period), the rates obtained in the current study are more or less in line with the broad range of estimates (41-70\%) derived in prior investigations, which have used the same measure (CFTQ) among adult British subjects with CF. ${ }^{5,6,11}$ Self-reported adherence rates in Brazilian samples have been somewhat higher $(60-84 \%) .^{12,28}$ The clinical threshold for sufficient adherence or persistence to ACT remains unclear. Ofcourse, our findings would be even more disquieting if we had used more conservative criteria for classifying persistence (ie, only those who "always" used ACT: $21.2 \%)$. In short, it appears that concerns expressed in previous studies are more salient when one considers patterns of ACT use over longer periods of time.

Identifying modifiable risk factors for poor persistence to ACT is an important priority for CF clinics. In the current study, bivariate analyses suggested that lower selfconfidence for performing ACT in the face of common barriers, greater concerns about ACT, and lower perceived necessity were each associated with poorer persistence over time. In multivariable analyses, self-efficacy and perceived concerns were the most robust predictors. Interestingly, similar findings emerged when we examined predictors of the point-prevalence of adherence at 12 months (as a continuous variable) rather than persistence over time, although the effects of concerns were only marginally significant. Overall, these results regarding self-efficacy (ie, confidence to manage obstacles) and outcome expectations (ie, anticipated favorable and unfavorable consequences) are consistent with social cognitive theory and with empirical findings in other patient populations outside of CF. ${ }^{14,15,17,18}$ They also seem consistent with findings from a recent study of adults with $\mathrm{CF}$, which found that self-efficacy and outcome expectancies were related to adherence to medication (ACT adherence was not evaluated). ${ }^{19}$ Self-efficacy and outcome expectations regarding specific treatments appear to offer pragmatic, modifiable targets for intervention.

Contrary to expectations, we found little support for the role of subjective norms regarding ACT. It seems that subjects' perceptions of social influence are not very important in shaping persistent ACT use. It is also possible that the single-item measure used in this study was not sufficiently reliable or sensitive, although such measures have been used successfully in other populations. Additional research would help clarify their relevance.

In contrast to the role of social cognitive factors (particularly self-efficacy and concerns) in predicting persistence, there was limited evidence for the importance of other subject variables that were assessed in exploratory analyses. There were hints that closer family ties and fewer negative interactions (social constraints) at baseline might be related to greater persistence. These particular aspects of social support have not been examined previously among adult subjects with $\mathrm{CF}$, and they might offer a meaningful direction for future research. In particular, it would be useful to examine dimensions of support specifically related to adherence (eg, emotional encouragement for adherence, instrumental assistance with ACT procedures, nagging and criticism). We did not find reliable relationships between depressive symptoms and subsequent persistence. Depressive symptoms were tied to poorer adherence in 2 previous investigations among adults with $\mathrm{CF}$, but these focused on medications rather than ACT. ${ }^{19,29}$ Perhaps these variables play a stronger role in predicting adherence for some subjects than others (eg, depression among subjects 


\section{Persistent Adherence to Airway Clearance Therapy}

with less family support). It would be interesting to examine these moderating or interaction effects in larger samples.

Generally, interventions designed to improve adherence in subjects with chronic illnesses have met with relatively modest effects. ${ }^{30}$ Few studies have addressed ACT adherence among adults with CF. Preliminary findings regarding quality improvement initiatives, ${ }^{31}$ mobile health interventions, ${ }^{32}$ and motivational interviewing ${ }^{33}$ have been promising, although few efforts have been explicitly theory-based or have evaluated mechanisms or processes of change. Recently, the National Institutes of Health Science of Behavior Change program has called on investigators to focus more directly on mechanisms of change in adherence behavior ${ }^{34}$; the social cognitive variables identified here appear to fit well with those recommendations. To help move the field forward, it may be useful to test whether proposed interventions create the expected shifts in these or other hypothesized mediating constructs thought to lie on the causal pathway to improved adherence and enhanced disease outcomes.

Strengths of the current investigation included the longitudinal research design, the clear theoretical foundation, and the use of validated measures. An obvious shortcoming is the reliance on self-report to assess adherence and persistence, and the limited number of assessment periods. There is no accepted standard for these evaluations, and different approaches have countervailing advantages and drawbacks. We used validated measures of adherence, which were carefully designed to minimize demand characteristics; moreover, persistence was not significantly related to a measure of social desirability response bias. Nonetheless, it is understood that the rates reported here underestimate nonadherence and nonpersistence. In future studies, more frequent assessments using multimodal approaches such as mobile daily diaries, ecological momentary monitoring, digital tracking, or proxy ratings would be useful. Another limitation is that this study included no information regarding the specific types of ACT that subjects were using; the analyses were not powered to examine these differences. Because patient preferences may vary across types of ACT (eg, chest physiotherapy, autogenic drainage, postural drainage and percussion, high-frequency chest wall oscillation), it would be helpful to evaluate the possibility of differences in persistence among these subgroups.

The study included adult subjects with $\mathrm{CF}$ from a rural southern region of the United States, and thus it is unclear how findings would generalize to other settings. Consistent with the population served by the CF clinic, the sample was predominantly white and highly diverse with respect to socioeconomic status; many of these young adults were economically disadvantaged. Health literacy was not assessed, but our clinical impression is that our subjects tend to be sophisticated about their illness. Notably, however, subjects with less education were more likely to be lost to follow-up; additional studies are needed that examine persistence, and its determinants, among subjects from diverse educational, cultural, and geographical backgrounds.

\section{Conclusions}

Our findings address an important gap in the literature by offering an initial picture of persistent adherence to ACT over time among adults with CF. Variables derived from Social Cognitive Theory, especially limited confidence in one's ability to engage in ACT in the face of daily obstacles, and greater concerns about ACT, represent pragmatic areas for screening and intervention efforts.

\section{REFERENCES}

1. Hoo ZH, Daniels T, Wildman MJ, Teare MD, Bradley JM. Airway clearance techniques used by people with cystic fibrosis in the UK. Physiotherapy 2015;101(4):340-348.

2. O'Donohoe R, Fullen BM. Adherence of subjects with cystic fibrosis to their home program: a systematic review. Respir Care 2014;59(11): 1731-1746.

3. Narayanan S, Mainz JG, Gala S, Tabori H, Grossoehme D. Adherence to therapies in cystic fibrosis: a targeted literature review. Expert Rev Respir Med 2017(2):11:129-145.

4. Macdonald M, Martin-Misener R, Helwig M, Smith LJ, Godfrey CM, Curran J, Murphy A. Experiences of adults with cystic fibrosis in adhering to medication regimens: a qualitative systematic review. JBI Database System Rev Implement Rep 2016(5):14:258-285.

5. Abbot J, Dodd M, Bilton D, Webb AK. Treatment compliance in adults with cystic fibrosis. Thorax 1994;49(2):115-120.

6. Conway SF, Pond MN, Hamnett T, Watson A. Compliance with treatment in adult patients with cystic fibrosis. Thorax 1996;51(1): 29-33.

7. Myers LB, Horn SA. Adherence to chest physiotherapy in adults with cystic fibrosis. J Health Psychol 2006;11(6):915-926.

8. Modi AC, Cassedy AE, Quittner AL, Accurso F, Sontag M, Koenig JM, Ittenbach RF. Trajectories of adherence to airway clearance therapy for patients with cystic fibrosis. J Pediatr Psychol 2010; 35(9):1028-1037.

9. Sontag MK, Quittner AL, Modi AC, Koenig JM, Giles D, Oermann $\mathrm{CM}$, et al. Investigators and Coordinators of the Airway Secretion Clearance Trial. Lessons learned from a randomized trial of airway secretion clearance techniques in cystic fibrosis. Pediatr Pulmonol 2010;45(3):291-300.

10. Abbot J, Dodd M, Webb AK. Health perceptions and treatment adherence in adults with cystic fibrosis. Thorax 1996;51(12):12331238.

11. White D, Stiller K, Haensel N. Adherence of adult cystic fibrosis patients with airway clearance and exercise regimens. J Cystic Fibrosis 2007;6(3):163-170.

12. Flores JS, Teixeira FA, Rovedder PME, Ziegler B, Dalcin PTR. Adherence to airway clearance therapies by adult cystic fibrosis patients. Respir Care 2013;58(2):279-285.

13. Abbott J, Dodd M, Gee L, Webb K. Ways of coping with cystic fibrosis: implications for treatment adherence. Disability Rehab 2001; 23(8):315-324. 


\section{Persistent Adherence to Airway Clearance Therapy}

14. Bandura A. Health promotion by social cognitive means. Health Educ Behav 2004;31(2):143-164.

15. Holmes EA, Hughes DA, Morrison VL. Predicting adherence to medications using health psychology theories: a systematic review of 20 years of empirical research. Value Health 2014;17(8):863-876.

16. Risser J, Jacobson TA, Kripalani S. Development and psychometric evaluation of the Self-Efficacy for Appropriate Medication Use Scale (SEAMS) in low-literacy patients with chronic disease. J Nurs Measurement 2007;15(3):203-219.

17. Horne R, Chapman SC, Parham R, Freemantle N, Forbes A, Cooper V. Understanding patients' adherence-related beliefs about medicines prescribed for long-term conditions: a meta-analytic review of the Necessity-Concerns Framework. PLoS One 2013;8(12):e80633.

18. Foot H, La Caze A, Gujral G, Cottrell N. The necessity-concerns framework predicts adherence to medication in multiple illness conditions: a meta-analysis. Patient Educ Couns 2016;99(5):706-717.

19. Hilliard ME, Eakin MN, Borrelli B, Green A, Riekert KA. Medication beliefs mediate between depressive symptoms and medication adherence in cystic fibrosis. Health Psychol 2015;34(5):496-504.

20. Bucks RS, Hawkins K, Skinner TC, Horn S, Seddon P, Horne R. Adherence to treatment in adolescents with cystic fibrosis: the role of illness perceptions and treatment beliefs. J Pediatric Psychol 2009; 34(8):893-902.

21. Ajzen I. Perceived behavioral control, self-efficacy, locus of control, and the theory of planned behavior. J Appl Soc Psychol 2002;32(4): 665-683.

22. Horne R, Weinman J, Hankins M. The Beliefs about Medicines Questionnaire (BMQ): the development and evaluation of a new method for assessing the cognitive representation of medication. Psychol Health 1999;14(1):1-24.

23. Courneya KS, Friedenreich CM. Utility of the theory of planned behavior for understanding exercise during breast cancer treatment. Psycho-Oncol 1999;8(2):112-122.

24. Reynolds WM. Development of reliable and valid short forms of the Marlowe-Crowne Social Desirability Scale. Clin Psychol 1982;38(1): 119-125.
25. Bjelland I, Dahl AA, Haug TT, Neckelmann D. The validity of the Hospital Anxiety and Depression Scale: an updated literature review. J Psychosom Res 2002;52(2):69-77.

26. Sherbourne CD, Kamberg CJ. Social functioning: Family and marital functioning measures. In: Stewart AL, Ware JE, editors. Measuring functioning and well-being: the medical outcomes study approach. Durham, NC; Duke University Press; 1992:182-193.

27. Lepore SJ, Silver RC, Wortman CB, Wayment HA. Social constraints, intrusive thoughts, and depressive symptoms among bereaved mothers. J Pers Soc Psychol 1996;70(2):271-282.

28. Dalcin PTR, Rampon G, Pasin LR, Ramon GM, Abrahao CLO, Oliveira VZ. Adherence to treatment in patients with cystic fibrosis. J Bras Pneumol 2007;33(6):663-670.

29. Knudsen KB, Pressler T, Mortensen LH, Jarden M, Skov M, Quittner AL, et al. Associations between adherence, depressive symptoms and health-related quality of life in young adults with cystic fibrosis. SpringerPlus 2016;5(1):1216.

30. Conn VS, Ruppar TM. Medication adherence outcomes of 771 intervention trials: systematic review and meta-analysis. Prev Med 2017;99:269-276.

31. Zanni RL, Sembrano EU, Du DT, Marra B, Bantang R. The impact of re-education of airway clearance techniques (REACT) on adherence and pulmonary function in patients with cystic fibrosis. BMJ Qual Saf 2014;23(Suppl 1):i50-i55.

32. Marciel KK, Saiman L, Quittell LM, Dawkins K, Quittner AL. Cell phone intervention to improve adherence: cystic fibrosis care team, patient, and parent perspectives. Pediatr Pulmonol 2010;45(2):157164.

33. Duff AJ, Latchford GJ. Motivational interviewing for adherence problems in cystic fibrosis: evaluation of training healthcare professionals. J Clin Med Res 2013;5(6):475-480.

34. Edmondson D, Falzon L, Sundquist KJ, Julian J, Meli L, Sumner JA, Kronish IM. A systematic review of the inclusion of mechanisms of action in NIH-funded intervention trials to improve medication adherence. Behav Res Ther 2018;101:12-19.

This article is approved for Continuing Respiratory Care Education credit. For information and to obtain your CRCE

(free to AARC members) visit www.rcjournal.com

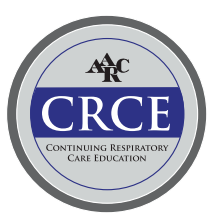

